\title{
Interactions of physico-chemical properties of the River Nile water and fungal diversity in River Nile streams in Delta region
}

\author{
Ahmed M. Abdel-Raheem, Gamalat A. Abd-Elaah, Osman M. El-Maghraby and Mahmoud S. \\ Bakhit \\ Botany department, Faculty of Science, Sohag University.
}

Rec. 20 Oct, 2018 Accept. 26 Nov, 2018

\begin{abstract}
Water pollution in Egypt is increasing over time, with constant supply of pollutant effluents to the water system from several sources. Water samples were collected for hydrochemicalanalysis during four successive seasons from some irrigation canals and streams of River Nile in Delta region governorates.There are various correlations patterns between fungal communities and physico-chemical parameters.Air and water temperature weremajor factors that affect fungal distribution. Calcium and magnesium have inverse correlation on fungal diversity. There was no significant correlation of $\mathrm{SO}_{4}{ }^{2-}$ andNO ${ }_{2}^{-}$with distribution of freshwater fungi. Ammonia and phosphate encouraged fungal growth.Salinity played active role in determining fungal diversity and community composition. Changes in species composition were correlated positively with elevated concentrations of $\mathrm{NH}_{3}{ }^{+}$, and pHin some governorates.
\end{abstract}

Key word: Physico-chemical, populations, Nile delta, Freshwater fungi.

\section{Introduction:}

Fungi may be exposed to a wide variety of organic and inorganic pollutants in the environment. Pollutants may exhibit toxicity and cause changes in fungal community composition (Gadd 2007). The effect of pollutants on fungal population community size and composition is particularly difficult to assess. Environmental pollution might be expected to lead to both toxic (destructive) and enrichment disturbance on fungal populations (Wainwright 1988). The resultant degree of toxic disturbance will depend upon both toxicant concentration and its availability to the fungal population, as well as to the susceptibility of the individuals involved. Toxicants may be have a selectivelyeffect on a few fungal species, or amore generalized effect.

Freshwater fungi are considered a cosmopolitan and a diverse group (McLaughlin et al., 2001). Lignicolous freshwater fungi grow on submerged woody debris in freshwater streams, ponds and lakes (Goh and Hyde 1996, Wong et al., 1998). Lignicolous freshwater fungi play an important role in the decomposition of submerged wood by breaking down lignocelluloses and releasing nutrients whichare important in ecosystem functioning (Yuen et al., 1998, Bucher et al., 2004, Hyde et al., 2016).

Several studies reported the effect of pollutants on freshwater fungal communities (Tan and Lim 1984; Maltby and Booth 1991; Au et al., 1992; Bärlocher 1992a; Tsui et al., 2001; Luo et al., 2004). The fungal community structure greatly differs with the physico-chemical properties of the respective habitats, such as water flow (PatteeandChergui 1995; Baldy et al., 2002), nutrient concentrations (Gulis et al., 2004; Rankovic 2005), salinity (Hyde and Lee1995; Roache $e t$ al., 2006), temperature (Bärlocher et al., 2008; Raja et al., 2009) and depth (Wurzbacher et al., 2010). The ability of fungi to survive in 
the presence of potentially toxic metals depends on a number of biochemical and structural properties, including physiological and/or genetically adaptation (Gadd 2000, 2007) .In general terms, toxic metals may affect fungal populations by reducing abundance and species diversity (Babich and Stotzky 1985; Arnebrant et al., 1987).

Water pollution in Egypt is increasing over time, with constant supply ofpollutant effluents to the water system from several sources. One of the major sources is the disposal of untreated or semi-treated domestic wastes into water bodies. The low level of sanitation service especially in rural areasmakes nearby streams (either canals or drains) the perfect places for inhabitants to dispose their sewage. Moreover, the excessive use of fertilizers and pesticides is another major source of water pollution. The effect of agricultural pollution was increased due to the extensive drainage reuse within the Nile Delta. Several studies revealed that untreated industrial wastes of more than 350 factories were discharged directly into the Nile and the Mediterranean Sea, most of them released explicitly known toxic and hazardous chemicals such as detergents, heavy metals and pesticides (RNPD 1989).

\section{Materials and Methods:}

Water samples were collected in clean oneliter polyethylene bottles for hydrochemicalanalysis during four successive seasons from irrigation canals and the River Nilein Delta region, during the period between February 2010 and December 2011.

Submerged, dead woody debris were collected randomly from irrigation canals and the River Nile, four times over 2 years. Sampling times included the four seasons (winter, summer, spring, and autumn) in the eight governorates (Dakahlya, Sharqiyah, Qalyubiya, Kafr El-Sheikh, El-Behera, Gharbeyia, Menofya and Damietta) within the Nile Delta region.

Chemical analysis of the collected water samples was carried out according to the standard methods (Rainwater et al., 1960;
Booth 1983). Fungal species richness, number of fungal collections per sample and number of records for each taxonomic group in the studied governorate and season collection were calculated. Canonical Correlation Analysis (CCA) was employed to test if variation in community composition is explained by physicho-chemical parameters.

\section{Hydrochemical analysis:}

Air and water temperature at each site were measured insitu using Celsius-thermometer. Potential of Hydrogen $(\mathrm{pH})$ values of water samples were measured using a digital $\mathrm{pH}$ meter (HANNA pH 211 microprocessor $\mathrm{pH}$ meters).

Electrical conductivity $(\mathrm{EC} \mu \mathrm{s} / \mathrm{cm})$ of water samples was measured using EC meter (model: HANNA HI 99300 Conductivity meter). Salinity, total dissolved solids (TDS) in $\mathrm{mg} / \mathrm{l}$ were measured for each sample using (HANNA HI 99300 Conductivity meter).Alkalinity was determined by titration using phenolphthalein and methyl orange indicators.

Chloride $\left(\mathrm{Cl}^{-}\right)$anion content was determined by volumetrically titration against silver nitrate using potassium chromate $\left(\mathrm{K}_{2} \mathrm{CrO}_{4}\right)$ as an indicator.Hardness was determined by titration of Erichrome black $\mathrm{T}$ indicator and standard $(0.01 \quad \mathrm{~N})$ Ethylenediaminetetraacetic acid (EDTA) solution. Calcium $\left(\mathrm{Ca}^{2+}\right)$ and Magnesium $\left(\mathrm{Mg}^{2+}\right)$ cations content of water samples were determined by complexometric titration using EDTA solution and Eriochrome black $T$ as indicator. Calcium was determined using (muroxide) indicator in presence of sodium hydroxide, while magnesium cation was calculated by subtracting the calcium value from $\left(\mathrm{Ca}^{2+}\right.$ and $\mathrm{Mg}^{2+}$ ) value after their determination using Eriochrome black T.

Sulphate $\left(\mathrm{SO}_{4}{ }^{2-}\right)$ was determined in water samples by the turbidimetric method. It is based upon the fact that barium sulphate tends to precipitate in a colloidal form. The absorbance of the barium sulphate solution is measured by turbidimeter and the sulphate ion concentration, determined by comparison of 
the reading with a standard curve.Ammonia $\left(\mathrm{NH}_{3}\right)$ concentration was determined in water samples using phenate method. Ammonia reacts with alkaline phenol and hypochlorite to form indophenol blue that is proportional to the ammonia concentration. The blue color is intensified with sodium nitroprusside to form a colored complex that absorbs at $660 \mathrm{~nm}$. Concentration of ammonia is determined by comparison of absorbance signal with calibration results obtained from prepared standards of varying concentrations (APHA 2005).

Phosohate $\left(\mathrm{PO}_{4}{ }^{3-}\right)$ anion concentration was determined colorimetrically using UV/visible spectrophotometer, at wavelength $700 \mathrm{~nm}$, using molybdate reagent and Tin chloride $\left(\mathrm{SnCl}_{2}\right)$ as indicator.

Nitrite $\left(\mathrm{NO}_{2}^{-}\right)$ion was determined by reaction of nitrite ions with sulfanylamide in acidic medium and the diazo compound obtained further reacts with diamine yielding in an azo color. The nitrite ion concentration is determined by measuring the absorbance of the azo color at 540-545 nm by spectrophotometer.

Statistical analysis: Variation in averages of physico-chemical parameters and fungi distribution among tested governorateswasanalysed using PAST softwareversion 2.11 (Hammer et al., 2001). Canonical correspondence analysis (CCA) included data of 218 fungal taxa and11 environmental parameters across 8 sampling governorates during 4 season's period was applied to evaluate role ofenvironmental variables in fungal communities.

\section{Results:}

Physical and chemical parameters varied between sites, governorates and seasons. Interaction between physico-chemical properties and diversity of freshwater fungi was strongly observed. The minimum, maximum and average values of hydrochemical analysis of the collected water sampleswere listed in table 1 . The patterns of their variations along the studied area and during study period are illustrated in figs. 1 to 7.

\section{Physico-chemical characteristics of River Nile water:}

The air temperature ranged between 16 and $44{ }^{0}$ Cduring the studied period. The maximum air temperature, $44{ }^{\circ} \mathrm{C}$, was recorded in August 2010 while the minimum temperature was recorded in December 2011 (Table 1). The water temperature ranged from 10 to $29{ }^{\circ} \mathrm{C}$. The highest water temperature was $29{ }^{\circ} \mathrm{C}$ in August 2010 while the lowest was $10{ }^{\circ} \mathrm{C}$ in December 2011(Table 1).Potential of Hydrogen $(\mathrm{pH})$ values ranged from 6 to 6.8 in the present study (Table 2).

Total dissolved salts(TDS) content of water samples were fluctuated between 227 ppm and614 ppm. The highest values of TDS were reported inKafr El-Sheikh (375), Damietta (328) and Sharqiyah (313) governorates, whereas the lowest ones occurred in Gharbeyia and Menofya governorates(Tables 1, 2 and Figure 1).

Electrical conductivity (EC) values exhibited great variationsbetween the different governorates and seasons and were fluctuated between $324 \mu \mathrm{s} / \mathrm{cm}$ and $932 \mu \mathrm{s} / \mathrm{cm}$ in the different sites in Nile Delta region. The highest averages of EC were reported fromKafr El-Sheikh (583), Sharqiyah (529) and Menofya (502). Alkalinity of studied streams and irrigation canals in the different four seasons was ranged between 88and 320mg/L. Kafr El-Sheikh and El Behera governorates had the highest values of alkalinity (Table 1).

Chloride concentrations ranged between 15 and $90 \mathrm{ppm}$ (Table 1). The highest values of chloride concentration were reported in Menofya and Qalyubiya governorates. In August 2010 collection (28-90ppm) whilethe lowest values in February 2010 (15-23ppm). Calcium and magnesium concentrations ranged between 25 and 57.5ppm, 13.8and 31.8 ppm,respectively. The average values of calcium and magnesium concentrations showednarrowdifferences between governorates and seasons collections. Nitrite 
concentration in water samples was fluctuated between 0.015 and $0.15 \mathrm{ppm}$ while, Ammonia concentrations ranged between 0.01 and $1.76 \mathrm{ppm}$ in the studied sites as shown in Table (1). Sulphateion concentrations ranged between 19.6 and $46 \mathrm{ppm}$. Meanwhile phosphate was in the range from 0.001 to 0.39 ppm (Table 1). The values of the physical and chemical parameters of the different seasons and governorates were listedin tables 2 and 3.
Some physico-chemical parameters exhibited strong peak in some studied governorates or seasons. For example, $\mathrm{NH}_{3}{ }^{+}$andCl-concentration werevery high in Menofya and Qalyubiya governorates and August collection, TDS peaked in KafrElSheikh and Damietta and also $\mathrm{Ca}{ }^{2+}$ peakedin KafrEl-Sheikh.

\begin{tabular}{|c|c|c|c|c|c|c|c|c|c|c|c|c|c|c|c|}
\hline \multirow{3}{*}{ Site } & \multicolumn{15}{|c|}{ February 2010 collection } \\
\hline & \multicolumn{5}{|c|}{ Physical properties } & \multicolumn{8}{|c|}{ Chemical properties } & \multicolumn{2}{|c|}{$\begin{array}{c}\text { Fungal } \\
\text { population }\end{array}$} \\
\hline & $\begin{array}{l}\text { A.T } \\
\text { OC }\end{array}$ & $\begin{array}{l}\text { W.T } \\
\text { OC }\end{array}$ & $\mathrm{pH}$ & $\begin{array}{l}\text { TDS } \\
\text { ppm }\end{array}$ & $\underset{\mu \mathrm{S} / \mathrm{cm}}{\mathrm{EC}}$ & $\begin{array}{c}\text { Alkalinity } \\
\text { mg/L }\end{array}$ & \begin{tabular}{c|c|}
$\mathbf{C l}$ \\
$\mathbf{p p m}$
\end{tabular} & \begin{tabular}{|l|}
$\mathrm{Ca}^{2+}$ \\
$\mathbf{p p m}$ \\
\end{tabular} & $\begin{array}{l}\mathrm{Mg}^{2+} \\
\mathbf{p p m}\end{array}$ & $\begin{array}{l}\mathrm{SO}_{4}{ }^{2-2} \\
\mathrm{ppm}\end{array}$ & $\begin{array}{l}\mathrm{NO}_{2}^{-} \\
\mathbf{p p m}\end{array}$ & $\begin{array}{l}\mathrm{NH}_{3} \\
\mathbf{p p m}\end{array}$ & $\begin{array}{l}\mathrm{PO}_{4}{ }^{3-} \\
\mathrm{ppm}\end{array}$ & S.R & NRS \\
\hline Dakahlya & \multirow{8}{*}{ 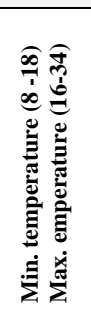 } & \multirow{8}{*}{$\stackrel{\infty}{\underline{b}}$} & 7.5 & 307.75 & 479.3 & 161.3 & 16.5 & 39.25 & 17 & 31.49 & 0.024 & 0.33 & 0.22 & 42 & 0.67 \\
\hline Sharqiyah & & & 6.9 & 227 & 442 & 130 & 15 & 34 & 15.4 & 35.8 & 0.009 & 0.023 & 0.09 & 31 & 1.12 \\
\hline Qalyubiya & & & 6.5 & 393.75 & 626.5 & 206.5 & 23.25 & 52.25 & 22.75 & 36.87 & 0.022 & 0.97 & 0.32 & 43 & 0.90 \\
\hline Kafr El-Sheikh & & & 6.6 & 281 & 480 & 104 & 17 & 32 & 19.2 & 29.7 & 0.032 & 0.037 & 0.12 & 20 & 0.90 \\
\hline El Behera & & & 6.7 & 408.5 & 650 & 247 & 22.5 & 52 & 22 & 40.5 & 0.041 & 0.46 & 0.06 & 23 & 0.82 \\
\hline Gharbeyia & & & 7.4 & 258 & 411.5 & 126.5 & 14.5 & 33 & 18 & 30.5 & 0.0015 & 0.045 & 0.015 & 30 & 0.73 \\
\hline Menofya & & & 6.9 & 280 & 446 & 88 & 15 & 36 & 27 & 32 & 0.003 & 0.6 & 0.11 & 24 & 1.24 \\
\hline Damietta & & & 6.8 & 310 & 498 & 112 & 19 & 40 & 22 & 33 & 0.044 & 0.04 & 0.07 & 20 & 0.95 \\
\hline Average & & & 6.9 & 308.25 & 504.16 & 146.91 & \begin{tabular}{|l|}
17.84 \\
\end{tabular} & 39.81 & 20.42 & 33.73 & 0.02 & 0.31 & 0.13 & 29 & 0.92 \\
\hline \multirow[t]{2}{*}{ Range } & & & $6.5-7.5$ & $227-408$ & 411-650 & $88-247$ & $15-23$ & $32-52$ & $15-27$ & \begin{tabular}{|l|}
$29.7-40.5$ \\
\end{tabular} & $.0015-.044$ & \begin{tabular}{|l|}
$.023-0.97$ \\
\end{tabular} & $.015-.32$ & $20-43$ & $0.67-1.24$ \\
\hline & & & \multicolumn{13}{|c|}{ August 2010 collection } \\
\hline Dakahlya & \multirow{8}{*}{ 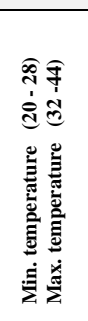 } & \multirow{8}{*}{ તิ } & 6.5 & 255.8 & 388.7 & 192.3 & 23.0 & 37.0 & 21.0 & 25.5 & 0.05 & 1.05 & 0.15 & 58 & 1.07 \\
\hline Sharqiyah & & & 6.1 & 463.7 & 703.67 & 247.7 & 45.0 & 50.33 & 27.13 & 35.9 & 0.03 & 0.92 & 0.07 & 37 & 1.02 \\
\hline Qalyubiya & & & 6.6 & 274.7 & 434 & 185.7 & \begin{tabular}{|l|}
55.67 \\
\end{tabular} & 45.13 & 20.37 & 31.13 & 0.08 & 1.31 & 0.21 & 39 & 0.83 \\
\hline Kafr El-Sheikh & & & 6.3 & 614.5 & 932.0 & 320.0 & 48.0 & 57.5 & 31.8 & 19.85 & 0.08 & 0.88 & 0.39 & 38 & 1.27 \\
\hline El Behera & & & 6.3 & 267 & 406 & 178 & 38 & 38 & 19 & 23 & 0.037 & 1.27 & 0.2 & 24 & 1.22 \\
\hline Gharbeyia & & & 6.2 & 237.5 & 360.5 & 137.5 & 31.5 & 29.0 & 19.5 & 35.5 & 0.07 & 1.76 & 0.02 & 44 & 1.12 \\
\hline Menofya & & & 6 & 303 & 460 & 105 & 90 & 40 & 16.8 & 22.5 & 0.08 & 1.5 & 0.2 & 35 & 1.78 \\
\hline Damietta & & & 6.7 & 414 & 577 & 244 & 28 & 44 & 24 & 23 & 0.019 & 0.68 & 0.09 & 23 & 0.80 \\
\hline Average & & & 6.3 & 353.78 & 532.73 & 201.3 & 44.9 & 42.62 & 22.45 & 27.05 & 0.06 & 1.17 & 0.17 & 37 & 1.14 \\
\hline \multirow[t]{2}{*}{ Range } & & & $6-6.7$ & $237-614$ & $360-932$ & $105-320$ & 23-90 & $25-57.5$ & \begin{tabular}{|l|}
$16.8-31.8$ \\
\end{tabular} & \begin{tabular}{|l|}
$19.8-35.9$ \\
\end{tabular} & $.019-.08$ & $.68-1.76$ & $.02-.39$ & 23-58 & $0.8-1.78$ \\
\hline & \multicolumn{15}{|c|}{ May 2011 collection } \\
\hline & \multicolumn{5}{|c|}{ Physical properties } & \multicolumn{8}{|c|}{ Chemical propertiess } & \multicolumn{2}{|c|}{$\begin{array}{c}\text { Fungal } \\
\text { population }\end{array}$} \\
\hline Site & $\begin{array}{l}\text { A.T } \\
\text { OC }\end{array}$ & $\begin{array}{l}\text { W.T } \\
\text { OC }\end{array}$ & pH & $\begin{array}{l}\text { TDS } \\
\text { ppm }\end{array}$ & $\begin{array}{c}\mathrm{EC} \\
\mu \mathrm{S} / \mathrm{cm}\end{array}$ & \begin{tabular}{|c|} 
Alkalinit \\
$\mathbf{y}$
\end{tabular} & $\begin{array}{c}\mathrm{Cl}^{-} \\
\mathbf{p p m}\end{array}$ & \begin{tabular}{l|}
$\mathbf{C a}^{2+}$ \\
$\mathbf{P p m}$
\end{tabular} & $\begin{array}{l}\mathrm{Mg}^{2+} \\
\mathbf{p p m}\end{array}$ & $\begin{array}{l}\mathrm{SO}_{4}^{2-} \\
\mathrm{ppm}\end{array}$ & $\begin{array}{l}\mathrm{NO}_{2}^{-} \\
\mathrm{ppm}\end{array}$ & $\begin{array}{l}\mathrm{NH}_{3} \\
\mathrm{ppm}\end{array}$ & $\begin{array}{l}\mathrm{PO}_{4}{ }^{3-} \\
\text { ppm }\end{array}$ & S.R & NRS \\
\hline Dakahlya & \multirow{8}{*}{ 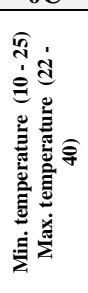 } & \multirow{8}{*}{$\begin{array}{l}\text { フิ } \\
\text { ঠे }\end{array}$} & 7.68 & 168.5 & 336.5 & 121 & 27.75 & \begin{tabular}{|l|}
27.0 \\
\end{tabular} & 16.4 & 22.18 & 0.01 & 0.09 & 0.15 & 56 & 1.21 \\
\hline Sharqiyah & & & 7.5 & 186 & 383 & 139 & 30 & 35 & 20 & 23.97 & 0.01 & 0.07 & 0.07 & 30 & 1.19 \\
\hline Qalyubiya & & & 7.2 & $\begin{array}{l}187.3 \\
\end{array}$ & 372.7 & 121.3 & 33.3 & 32.8 & 21.2 & 21.5 & 0.05 & 0.06 & 0.08 & 49 & 0.81 \\
\hline Kafr El-Sheikh & & & 7.6 & 244 & 410 & 141 & 34 & 45 & 23 & 35.7 & 0.06 & 0.15 & 0.06 & 24 & 1.23 \\
\hline El Behera & & & 7.5 & 180 & 360 & 133 & 29 & 30 & 17 & 24.4 & 0.025 & 0.025 & 0.09 & 30 & 1.09 \\
\hline Gharbeyia & & & 7.6 & 175 & 350 & 110 & 23 & 25.2 & 17 & 24.4 & 0.02 & 0.01 & 0.015 & 43 & 1.03 \\
\hline Menofya & & & 7.5 & 188 & 355 & 140 & 34 & 30 & 18 & 30.3 & 0.077 & 1.48 & 0.02 & 36 & 1.0 \\
\hline Damietta & & & 7.65 & 198.5 & 400 & 146.5 & 35 & 42 & 22.5 & 34.3 & 0.04 & 0.23 & 0.03 & 37 & 1.26 \\
\hline \begin{tabular}{|l|} 
Average \\
\end{tabular} & & & 7.46 & 184.3 & 367.67 & 128.5 & 31.2 & 32.5 & 19.53 & 25.16 & 0.03 & 0.21 & 0.07 & 38 & 1.10 \\
\hline Range & & & $7.2-7.7$ & 168-244 & $336-400$ & 110-1469 & 23-35 & $25-45$ & \begin{tabular}{|l|}
$16.4-23$ \\
\end{tabular} & 19.6-39.1 & $0.01-0.07$ & \begin{tabular}{|l|}
$0.01-1.48$ \\
\end{tabular} & 0.01-0.15 & $24-56$ & $0.8-1.26$ \\
\hline
\end{tabular}




\begin{tabular}{|c|c|c|c|c|c|c|c|c|c|c|c|c|c|c|c|}
\hline \multirow[b]{2}{*}{ Dakahlya } & \multirow{3}{*}{ EF } & & \multicolumn{13}{|c|}{ December 2011 collection } \\
\hline & & \multirow{8}{*}{$\frac{\mathfrak{d}}{\hat{\theta}}$} & 7.7 & 323 & 505 & 172 & 49 & 31 & 15.2 & 39 & 0.06 & 0.26 & 0.012 & 52 & 0.95 \\
\hline Sharqiyah & & & 7.8 & 377 & 589 & 193 & 55 & 36 & \begin{tabular}{ll|}
18.8 \\
\end{tabular} & 45 & 0.15 & 0.4 & 0.08 & 24 & 0.73 \\
\hline Qalyubiya & \multirow{6}{*}{ 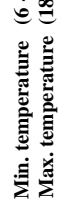 } & & 7.4 & 315 & 448 & 173 & 47 & 33 & 16 & 37 & 0.008 & 0.2 & 0.02 & 42 & 1.05 \\
\hline Kafr El-Sheikh & & & 7.3 & 364 & 510 & 207 & 31 & 35 & 20 & 35.7 & 0.04 & 0.25 & 0.08 & 24 & 0.85 \\
\hline El Behera & & & 7.4 & 380 & 460 & 213 & 39 & 31 & 19 & 24.4 & 0.02 & 0.035 & 0.06 & 15 & 0.76 \\
\hline Gharbeyia & & & 7.5 & 300 & 468 & 178 & 34 & 33 & 17.5 & 44 & 0.03 & 0.1 & 0.02 & 43 & 0.91 \\
\hline Menofya & & & 7.5 & 310 & 746 & 169 & 45 & 34 & 17.5 & 46 & 0.04 & 0.3 & 0.013 & 41 & 0.9 \\
\hline Damietta & & & 7.6 & 390 & 433 & 165 & 40 & 29 & 14.3 & 41 & 0.08 & 0.19 & 0.013 & 30 & 0.83 \\
\hline Average & & & 7.53 & 345 & 520 & 183.8 & 42.5 & 32.8 & 17.3 & 39.01 & 0.05 & 0.2 & 0.04 & 33.8 & 87.3 \\
\hline Range & & & 7.3-7.8 & \begin{tabular}{|l|}
$300-390$ \\
\end{tabular} & 433- 746 & $165-213$ & $31-55$ & $29-36$ & $14.3-20$ & $24.4-46$ & .008-0.15 & $0.03-0.4$ & $0.001-0.08$ & $15-52$ & $0.73-1.05$ \\
\hline
\end{tabular}

Table (1): The variation in physical and chemical parameters of the collected water samples from the eight governorates in different seasons.

A.T $=$ Air temperature; W.T $=$ Water temperature, $\mathrm{TDS}=$ Total dissolved salts: $\mathrm{EC}=$ Electric conductivity; $S . R=$ Species richness; $\mathbf{N R S}=$ Number of records per sample.

\begin{tabular}{|c|c|c|c|c|c|c|c|c|c|c|c|c|c|c|c|c|}
\hline \multirow[b]{2}{*}{ Site } & \multicolumn{4}{|c|}{ Physical properties } & \multicolumn{7}{|c|}{ Chemical properties } & \multicolumn{5}{|c|}{ Fungal records number } \\
\hline & pH & TDS & EC & Alkalinity & $\mathrm{Cl}^{-}$ & $\mathrm{Ca}^{2+}$ & $\mathbf{M g}^{2+}$ & $\mathrm{SO}_{4}{ }^{2-}$ & $\mathrm{NO}_{2}^{-}$ & $\mathbf{N H}_{3}$ & $\mathrm{PO}_{4}{ }^{3-}$ & NRS & As $\mathrm{R}$ & An R & B R & M R \\
\hline Dakahlya & 7.35 & 263.76 & 427.4 & 161.6 & 29.1 & 33.56 & 17.40 & 29.54 & 0.04 & 0.43 & $\mathbf{0 . 1 3}$ & 0.93 & 396 & 246 & 17 & 58 \\
\hline Sharqiyah & 7.08 & 313.43 & 529.4 & 177.4 & 36.3 & 38.83 & 20.33 & 35.17 & 0.05 & 0.35 & 0.08 & 1.07 & 182 & 97 & 11 & 47 \\
\hline Qalyubiya & 6.93 & 292.69 & 470.3 & 171.6 & 39.8 & 40.8 & 20.08 & 31.63 & 0.04 & 0.64 & 0.16 & 0.91 & 264 & 200 & 10 & 51 \\
\hline $\begin{array}{ll}\text { Kafr } \\
\text { Sheikh }\end{array} \quad$ El- & 6.95 & 375.88 & $\mathbf{5 8 3 . 0}$ & 193.0 & 32.5 & 42.38 & 23.50 & 30.24 & 0.05 & 0.33 & 0.16 & 1.19 & 144 & 111 & 5 & 63 \\
\hline El Behera & 6.98 & 308.88 & 469.0 & 192.7 & 32.1 & 37.75 & 19.25 & 28.08 & 0.03 & 0.45 & 0.10 & 1.02 & 135 & 69 & 3 & 30 \\
\hline Gharbeyia & 7.18 & 242.63 & 397.5 & 138.0 & 25.7 & 30.05 & 18.00 & 33.60 & 0.03 & 0.48 & 0.02 & 0.96 & 295 & 185 & 9 & 50 \\
\hline Menofya & 6.98 & 270.25 & 501.7 & 125.5 & 46.0 & 35.00 & 19.83 & 32.70 & 0.05 & 0.97 & 0.09 & 1.2 & 178 & 144 & 9 & 17 \\
\hline Damietta & 7.19 & 328.13 & 477.0 & 166.9 & 30.5 & 38.75 & 20.70 & 32.83 & 0.05 & 0.29 & 0.05 & 1.04 & 204 & 102 & $\mathbf{0}$ & 12 \\
\hline
\end{tabular}

Table (2): The correlation between average of physico-chemical parameters and the fungal recordsnumbers in the studied governorates.

\begin{tabular}{|c|c|c|c|c|c|c|c|c|c|c|c|c|c|c|c|c|c|c|c|}
\hline \multirow[b]{2}{*}{ Seasons } & \multicolumn{7}{|c|}{ Physical properties } & \multicolumn{7}{|c|}{ Chemical propertiess } & \multicolumn{5}{|c|}{ Fungal records number } \\
\hline & \begin{tabular}{|c|} 
Min \\
A.T ${ }^{0} \mathrm{C}$
\end{tabular} & $\begin{array}{c}\text { Max } \\
\text { A.T }{ }^{0} \mathrm{C}\end{array}$ & $\begin{array}{l}\text { W.T } \\
{ }^{0} \mathbf{C}\end{array}$ & pH & $\begin{array}{l}\text { TDS } \\
\text { ppm }\end{array}$ & $\begin{array}{c}\mathrm{EC} \\
\mu \mathrm{S} / \mathrm{cm}\end{array}$ & Alkalinity & $\begin{array}{c}\mathrm{Cl}^{-} \\
\mathbf{p p m}\end{array}$ & $\begin{array}{l}\mathbf{C a}^{2+} \\
\mathbf{P p m}\end{array}$ & $\left|\begin{array}{l}\mathbf{M g}^{2+} \\
\mathbf{p p m}\end{array}\right|$ & $\begin{array}{l}\mathrm{SO}_{4}{ }^{2-} \\
\mathrm{ppm}\end{array}$ & $\begin{array}{l}\mathrm{NO}_{2}^{-} \\
\mathbf{p p m}\end{array}$ & $\begin{array}{l}\mathrm{NH}_{3} \\
\mathrm{ppm}\end{array}$ & $\begin{array}{l}\mathrm{PO}_{4}{ }^{3-} \\
\text { ppm }\end{array}$ & NRS & As R & An $\mathbf{R}$ & B R & $\mathbf{M} \mathbf{R}$ \\
\hline $\begin{array}{l}\text { February } 2010 \\
\text { (winter) }\end{array}$ & 8-18 & $16-34$ & 16-18 & 6.9 & 308.3 & 504.2 & 146.9 & 17.84 & 39.8 & 20.4 & 33.7 & 0.02 & 0.31 & 0.13 & 0.89 & 311 & 285 & 14 & 98 \\
\hline $\begin{array}{l}\text { August } 2010 \\
\text { (summer) }\end{array}$ & 20-28 & $32-44$ & 27-29 & 6.3 & 353.8 & 532.7 & 201.3 & 44.9 & 42.6 & 22.5 & 27.1 & 0.06 & 1.17 & 0.17 & 1.1 & 463 & 262 & 23 & 138 \\
\hline
\end{tabular}




\begin{tabular}{|l|c|c|c|c|c|c|c|c|c|c|c|c|c|c|c|c|c|c|c|}
\hline $\begin{array}{l}\text { May 2011 } \\
\text { (last spring) }\end{array}$ & $10-25$ & $22-40$ & $20-22$ & 7.46 & 184.3 & 367.7 & 128.5 & 31.21 & 32.5 & 19.5 & 25.2 & 0.03 & 0.21 & 0.07 & 1.05 & 552 & 280 & 13 & 75 \\
\hline $\begin{array}{l}\text { December 2011 } \\
\text { (autumn) }\end{array}$ & $6-17$ & $18-24$ & $10-12$ & 7.5 & 345 & 520 & 183.8 & 42.5 & 32.8 & 17.3 & 39.0 & 0.05 & 0.2 & 0.04 & 1.0 & 472 & 327 & 14 & 17 \\
\hline
\end{tabular}

Table (3): Effect of average of physico-chemical and seasonal parameters on the number of fungal collections.

NRS = number of records per sample; As $\mathrm{R}=$ ascomycetes records, $\quad$ An $\mathrm{R}=$ anamorphic fungi record; $\mathrm{BR}=$ Basidiomycetesrecords; $\mathrm{MR}=$ Myxomycetes records.

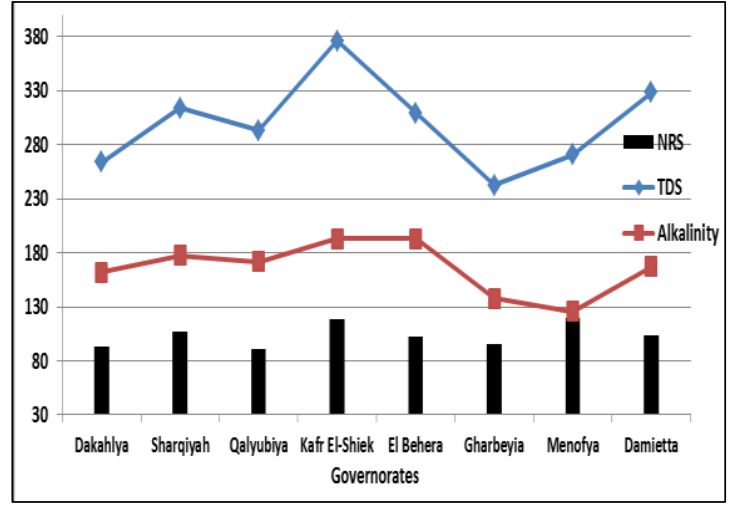

Fig. (1):The relation between number of fungal collections per sample andaverages of TDS, alkalinity in the eight studied governorates (NRS $x$ $\left.10^{2}\right)$.

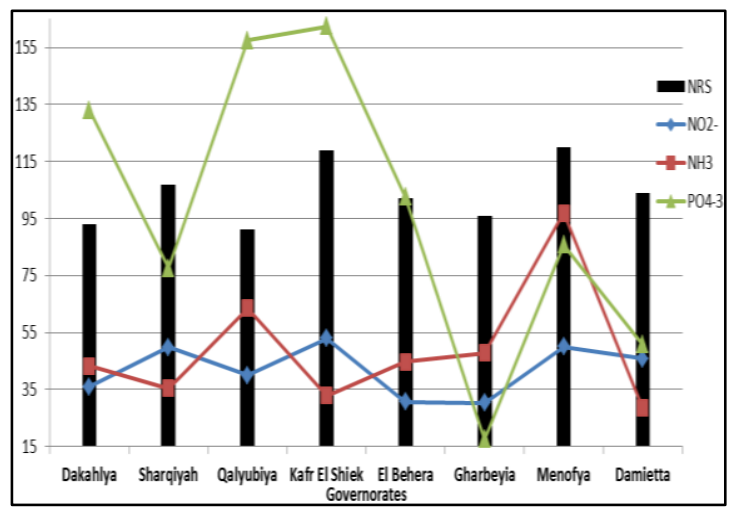

Fig. (2):The relation between numbers of records per sampleand averages of $\mathrm{NO}_{2}^{-}, \mathrm{NH}_{3}$ and $\mathrm{PO}_{4}{ }^{3-}$ ions in the studied governorates(NRS, $\mathrm{NO}_{2}^{-}, \mathrm{NH}_{3}$, $\left.\mathrm{PO}_{4}{ }^{3-} \times 10^{2}\right)$.

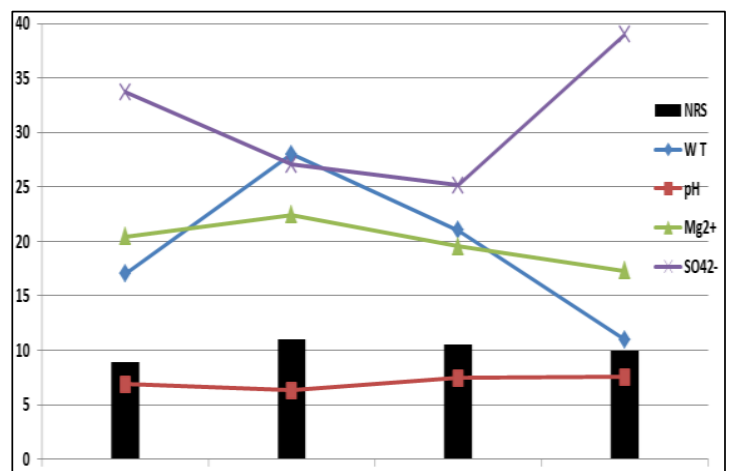

Fig. (3): The relation between numbers of records per sampleandaverages ofwater temperature, $\mathrm{pH}$, $\mathrm{Mg}^{2+}, \mathrm{SO}_{4}{ }^{2-}$ in the studied seasons (NRS x 10).

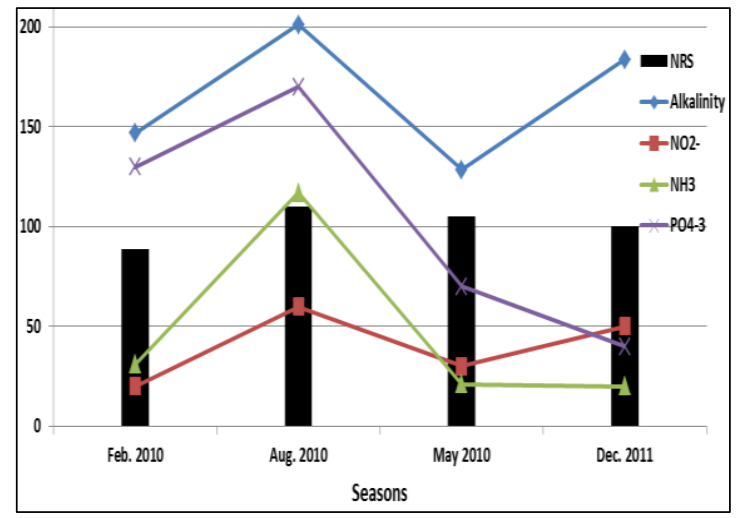

Fig. (4): The relation between numbers of records per sampleandaverages ofalkalinity, $\mathrm{NO}_{2}{ }^{-}, \mathrm{NH}_{3}$ and $\mathrm{PO}_{4}{ }^{3-}$ ions in the studied seasons (NRS, $\mathrm{NO}_{2}{ }^{-}$, $\mathrm{NH}_{3}, \mathrm{PO}_{4}{ }^{3-} \times 10^{2}$ ).

The relationship between fungal distribution parameters and physichochemical analysis:

By using direct observation to the results, we cannot put constant base or direct correlation betweenphysicho-chemical analysis and biodiversity of fungi for example: the highest number of records per sample was recorded in Menofya governorate in August collectionand also the highest average of $\mathrm{NO}_{2}{ }^{-}$ and $\mathrm{NH}_{3}{ }^{+}$were recorded in the same governorate.Otherwise, approximately the 
lowest average of TDS, alkalinity and $\mathrm{Mg}^{2+}$ were recorded in Menofya governorate (Tables 2 and 3).The lowest number of records per samplein December 2011 was reported in Sharqiyahgovernorates, where the concentration of $\mathrm{Ca}^{+2}$ and $\mathrm{SO}_{4}{ }^{2-}$ was high.Many other examples of correlation patterns between physico-chemical parameters and fungal population were in Tables(1,2 and 3) and Figs. (1 to 4). In this study ascomycetes and anamorphic fungi records were high in governorates with low salinity(e.g. Dakahlya, Qalyubiya and Gharbeyia governorates). Some family like Halosphaeriaceae exhibited more preference to governorates with high total dissolved salts; Damietta, Sharqiyah and KafrEl-sheikh governorates.

We try to conclude clear relation between physico-chemical results and biodiversity of fungi using correspondence analysis (CCA). The environmental data were correlated with frequency of occurrence of fungal species data set, and the results for the CCA of governorates in relation to environmental variables were given in Table (4)and Fig. (5). Canonical correspondence analysis (CCA) triplot explained $41.6 \%$ of the variability in the fungal community and environmental data with $22.2 \%$ in axis 1 and $19.4 \%$ in axis 2 (Table 4). The orientation of the environmental axis reflects the direction of maximum change of that variable.The longer of the axis indicate to thestrengthofinfluenceof variable on community composition. Thus sites near or beyond the tip of an axis will be strongly positively correlated with and influenced by the environmental variable represented by that arrow. Axis 1 was negatively correlated with $\mathrm{PO}_{4}{ }^{3-}$ and $\mathrm{EC}$ variables Likewise, a significant positive correlation of axis 1 was observed with $\mathrm{pH}$ average values. A significant positive correlation of axis 2 with $\mathrm{NH}_{3}$ was observed, while it was significantly negatively correlated with alkalinity, TDS, $\mathrm{Ca}^{+2}$ and $\mathrm{Mg}^{2+}$ (Table 4 and Fig 5). There was no significant correlation of $\mathrm{SO}_{4}{ }^{2-}$ and $\mathrm{NO}_{2}{ }^{-}$with both axes.

\begin{tabular}{|c|c|c|}
\hline & \multicolumn{2}{|c|}{ CCA Axes } \\
\hline Variables & 1 & 2 \\
\hline Eigenvalue (\%) & $0.237(22.2 \%)$ & $0.207(19.4 \%)$ \\
\hline $\mathrm{pH}$ & 0.46 & 0.12 \\
\hline $\mathrm{TDS}$ & 0.01 & -0.77 \\
\hline $\mathrm{EC}$ & -0.33 & -0.45 \\
\hline Alkalinity & -0.08 & -0.86 \\
\hline $\mathrm{Cl}-$ & -0.29 & 0.50 \\
\hline $\mathrm{Ca}{ }^{+2}$ & -0.03 & -0.60 \\
\hline $\mathrm{Mg}^{2+}$ & -0.13 & -0.58 \\
\hline $\mathrm{SO}^{2-}$ & 0.11 & 0.14 \\
\hline $\mathrm{NO}^{2}$ & 0.11 & -0.07 \\
\hline $\mathrm{NH}_{3}$ & -0.30 & 0.87 \\
\hline $\mathrm{PO}_{4}{ }^{3-}$ & -0.42 & -0.20 \\
\hline \hline
\end{tabular}

Table 4:Summary of canonical correspondence analysis (CCA) using PAST software.

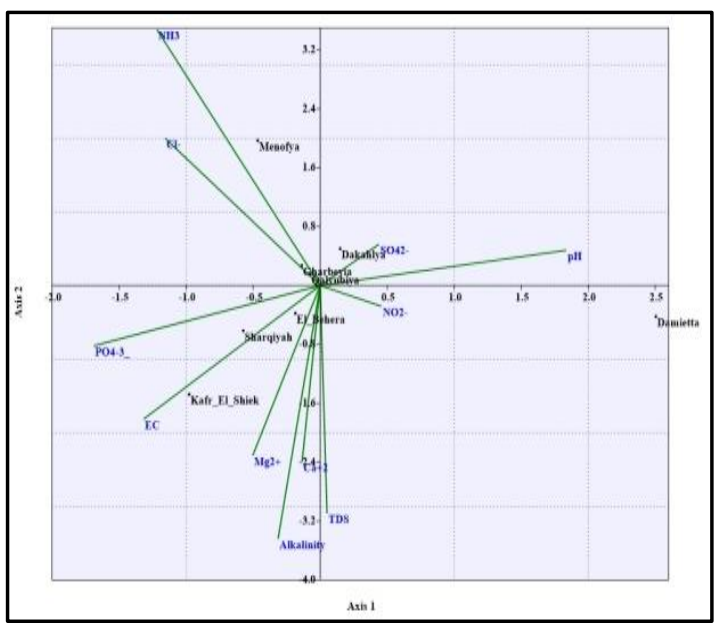

Fig. 5:Canonical correspondence analysis (CCA) plot explaining impact of environmental variables on species distribution

\section{Discussion:}

This part of the present investigation was planned to monitor the physico-chemical properties of water samples in river Nile streams in Delta region and study the effects of the changes in fungal distribution in the different governorates and seasons. The River 
Nile and its streams in Egypt can be characterized to high, moderately and low polluted. The Nile water is of high quality as the river reaches Cairo. Deterioration in water quality occurs when the Nile splits into the Damietta and Rosetta branches in a northward direction due to industrial effluents and agricultural drainage with decreasing flows (World Bank 2005).

The distribution pattern of fungi within streams can be profoundly altered by human activities, including the input of nutrients, sewage, pesticides and industrial wastes (Bärlocher 1992b). Factors affecting colonization of fungi involve types of substratum, temperature, relative humidity, the season of sampling, water chemistry and geographical location (Kohlmeyer and Kholmeyer 1979; Hudson 1986). Air and water temperature were ones of the main factors effecting on the fungal distribution between different seasons collections. The highest species richness and ascomycete records were observed in summer and May collections while anamorphic fungi records were high in February and December collections. Shearer (1972) and Suberkropp (1984) found that seasonal changes in temperature affected the occurrence of aquatic ascomycetes and hyphomycetes.

Chamier (1992) reviewed a number of important factors that effect on the fungal communities inside the stream such as water temperature, conductivity, $\mathrm{pH}$, nitrate and phosphorus concentration. Furthermore, $\mathrm{Hu}$ et al. (2010) suggested that changes in water aeration, $\mathrm{pH}$ and turbidity may be causal factors on fungal communities. In this investigation $\mathrm{pH}$ values was slightly fluctuated and not exceeded 7.8 and contrary correlated with most fungal distribution parameters in Nile Delta region. The $\mathrm{pH}$ value is particularly play a crucial role in determining the fungal diversity and their composition. Dubey et al. (1994) stated that the increase in water acidity lead to reduce the diversity of aquatic hyphomycetes in West Virginia. Also, Bärlocher and Rosset (1981) reported that the fungal diversity in soft water streams was higher than those in hard water streams where the $\mathrm{pH}$ was higher. The fluctuation in $\mathrm{pH}$ arises from different industrial effluents and agricultural drainage in waters.

Electric conductivity of Nile water samples exhibited great differences between the different governorates and different season's samples. Electric conductivity and alkalinity have a contrary effect on the most fungal distribution parameters in studied governorates and seasons. The increase in the EC was resulting from agricultural drainage which has high conductivity (APHA 1998; Gali et al. 2012).Salinity played active role in determining fungal diversity and community composition in this study. Less is known about the variation of fungal diversity and composition along latitude, altitude and salinity gradients (Mohamed and Martiny 2010). In general there was decrease in fungal diversity with increasing salinity in this study.

Ammonia and phosphate have enhancing effect in most distribution parameter in this investigation. The level of nitrates and phosphate in water has been regarded as important limiting factors in determining rates of detritus degradation (Elwood et al. 1981; Suberkropp and Chauvet 1995). Sridhar and Bärlocher (1997) found that the sporulation of aquatic hyphomycetes was stimulated by increasing nitrates. The presence or increase in $\mathrm{NO}_{2}^{-}$and $\mathrm{NH}_{3}{ }^{+}$in some streams may be resulting from fertilizer that may be present in the soil and it easily oxidized to nitrite (Karavoltsos et al. 2008). There was no significant correlation of $\mathrm{SO}_{4}{ }^{2-}$ and $\mathrm{NO}_{2}{ }^{-}$with fungi reported during this study.Suberkropp et al. (1988) found that the aquatic hyphomycetes community was unaffected by the effluents discharged from a sewage treatment plant.

Shearer (1972) studied the distribution of aquatic ascomycetes at 5 stations along river in Chesapeake Bay. She found that 6 species collected at station 1 and 3 were not collected at station 2 and suggested that was probably because of the effluents discharged from a 
sewage treatment plant near station 2 . Tsui et al. (2001) investigated the longitudinal and temporal distribution of freshwater ascomycetes and hyphomycetes on submerged wood in the Lam Tsuen River in Hong Kong, and found that the fungal species composition changed correlated with concentrations of $\mathrm{NO} 3-\mathrm{N}, \mathrm{NH}_{3}-\mathrm{N}$, and $\mathrm{PO}_{4}-\mathrm{P}$ resulting from human disturbance. Luo et al. (2004) compared the fungal communities and diversity between Lake Fuxian (an unpolluted lake) and Lake Dianchi (a heavily polluted lake), and concluded that pollution causes change in the fungal communities, but had little effect on fungal diversity. The number of fungal species in submerged wood may not be good bio- indicators of organic pollution (Tsui et al. 2001). Au et al. (1992) and Raviraja et al. (1998) compared the species diversity in unpolluted and polluted streams located geographically close together. They observed that freshwater fungi diversity, species richness and conidial production were reduced with organic pollution in the polluted streams.

$\mathrm{Ca}^{+2}$ and $\mathrm{Mg}^{2+}$ concentrationwere negatively correlated with fungal communities. However, physico-chemical parameters such as $\mathrm{SO}_{4}{ }^{2-}$ and $\mathrm{NO}_{2}$ they were not having significant correlation on fungal communities. Many metals e.g., copper $(\mathrm{Cu})$, magnesium $(\mathrm{Mg})$, potassium $(\mathrm{K})$, sodium $(\mathrm{Na})$, iron $(\mathrm{Fe})$ are essential to fungal growth, development and differentiation (Hughes and Poole 1991). However, metals can be toxic above a critical concentration that depends on the organism, the physico-chemical properties of the metal, and the environmental factors (Gadd 1993; Blaudez et al. 2000; Gimmler et al. 2001). Although fungal diversity has been recognized to decrease due to heavy metal exposure (Bermingham et al. 1996), several species of aquatic hyphomycetes have been found in metal-polluted streams (Krauss et al. 2001; Pascoal et al. 2005).

Fluctuation observedhereinphysichochemicalparametersare probably due to combination of several factors including industrial, agricultural and domestic waste discharge. Results from CCA analysis of the fungal assemblages found at the eight governorates revealed that changes in species composition were correlated with elevated concentrations of $\mathrm{NH}_{3}$, and $\mathrm{pH}$ resulting from domestic and agricultural activities in River Nile streams.

\section{References:}

American Public Health association, APHA (1998). Standard Methods for the Examination of Water and Wastewater, 20th ed., American Public Health Association, Washington, DC, USA.

American Public Health Association, APHA (2005). Standard Methods for Examinations of Water and Wastewater, 21st ed. AWWA and WEF DC, Washington.

Arnebrant, K., Baath, E. and Nordgren, A. (1987). Copper tolerance of microfungi isolated from polluted and unpolluted forest soil. Mycologia. 890-895.

$\mathrm{Au}$, D.W., Hodgkiss, I.J. and Vrijmoed, L.L. (1992). Fungi and Cellulolytic Activity Associated with Decomposition of Bauhinia-Purpurea Leaf Litter in a Polluted and Unpolluted Hong-Kong Waterway. Can. J Bot. 70 (5):1071-1079.

Babich, H. and Stotzky, G. (1985). Heavy metal toxicity to microbe-mediated ecologic processes: a review and potential application to regulatory policies. Environ. Res. 36 (1): 111137.

Baldy, V., Chauvet, E., Charcosset, J-Y and Gessner, M.O. (2002). Microbial dynamics associated with leaves decomposing in the mainstem and floodplain pond of a large river. Aquat. Microb. Ecol. 28 (1): 25-36.

Bärlocher, F. (1992a). Effects of Drying and Freezing Autumn Leaves on Leaching and Colonization by Aquatic Hyphomycetes. Freshwater Biol. 28 (1): 1-7. 
Bärlocher, F. (1992b). The ecology of aquatic hyphomycetes. Springer-Verlag, Berlin and Heidelberg, Germany. 225 pp.

Bärlocher, F. and Rosset, J. (1981). Aquatic hyphomycete spora of two Black Forest and two Swiss Jura streams. Trans. Brit. Mycol. Soc. 76 (3): 479483.

Bärlocher, F., Seena, S., Wilson, K.P. and Williams, D.D. (2008). Raised water temperature lowers diversity of hyporheic aquatic hyphomycetes. Freshwater Biol. 53 (2):368-379.

Bermingham, S., Maltby, L. and Cooke, R.C. (1996). Effects of a coal mine effluent on aquatic hyphomycetes. I. Field study. J. Appl. Ecol. 33: 1311-1321.

Blaudez, D., Jacob, C., Turnau, K., Colpaert, J.V., Ahonen-Jonnarth, U., Finlay, R., Botton, B. and Chalot, M. (2000). Differential responses of ectomycorrhizal fungi to heavy metals in vitro. Mycol. Res. 104: 1366-1371.

Bucher, V.V.C., Hyde, K.D., Pointing, S.B., Reddy, C.A. (2004). Production of wood decay enzymes, mass loss and lignin solubilization in wood by diverse freshwater fungi. Microbial Ecology 48, 331-337.

Chamier A-C (1992). Water chemistry. In: The ecology of aquatic hyphomycetes. Springer. 152-172.

Dubey, T., Stephenson, S.L. and Edwards, P.J. (1994). Effect of $\mathrm{pH}$ on the distribution and occurrence of aquatic fungi in six West Virginia mountain streams. J. Environ. Qual. 23 (6): 1271-1279.

Elwood, J.W., Newbold, J.D., Trimble, A.F. and Stark, R.W. (1981). The limiting role of phosphorus in a woodland stream ecosystem: effects of $\mathrm{P}$ enrichment on leaf decomposition and primary producers. Ecology. 62: 146158.

Gadd, G.M. (1993). Interactions of fungi with toxic metals. New Phytol. 124: 25-60.
Gadd, G.M. (2000). Microbial interactions with tributyltin compounds: detoxification, accumulation, and environmental fate. Sci. Total Environ. 258 (1):119-127.

Gadd, G.M. (2007).Geomycology: biogeochemical transformations of rocks, minerals, metals and radionuclides by fungi, bioweathering and bioremediation. Mycol. Res. 111:3-49.

Gali, R.K., Soupir, M.L. and Helmers, M.J. (2012). Electrical conductivity as a tool to estimate chemical properties of drainage water quality in the Des Moines Lobe, Iowa.

Gimmler, H., de Jesus, J. and Greiser, A. (2001). Heavy metal resistance of the extreme acidotolerant filamentous fungus Bispora sp. Microb. Ecol. 42: 87-98.

Goh, T.K., Hyde, K.D. (1996). Biodiversity of freshwater fungi. Journal of Industrial Microbiology \& Biotechnology 17: 328-345.

Gulis, V., Rosemond, A.D., Suberkropp, K., Weyers, H.S. and Benstead, J.P. (2004). Effects of nutrient enrichment on the decomposition of wood and associated microbial activity in streams. Freshwater Biol. 49 (11):1437-1447.

Hammer, O., Harper, D.A. and Ryan, P. (2001). Past: paleontological statistics software package for education and data analyses. Palaeontographica Electronica. 4 (1): pp.178.

Hu, D., Cai, L., Chen, H., Bahkali, A.H. and Hyde, K.D. (2010). Fungal diversity on submerged wood in a tropical stream and an artificial lake. Biodivers Conserv. 19 (13): 3799-3808.

Hudson, H.J. (1986). Fungi as inhabitants of extreme environments. In: Fungal Biology, Edward Arnold Publishers, Great Britain. 298 p.

Hughes, M.N. and Poole, R.K. (1991). Metal speciation and microbial growth the 
hard (and soft) facts. J. Gen. Microbiol. 137. (4):725-734.

Hyde, K.D. and Lee, S.Y. (1995). Ecology of Mangrove Fungi and Their Role in Nutrient Cycling-What Gaps Occur in Our Knowledge. Hydrobiologia. 295 (1-3): 107-118.

Hyde, K.D., Fryar, S., Tian, Q., Bahkali, A.H. et al. (2016). Lignicolous freshwater fungi along a north-south latitudinal gradient in the Asian/Australian region; can we predict the affects of global warming on biodiversity and function. Fungal Ecology 19, 190200.

Karavoltsos, S., Sakellari, A., Mihopoulos, N., Dassenakis, M. and Scoullos, M. (2008). Evaluation of the quality of drinking water in regions of Greece, Desalination. 224: 317-329.

Kohlmeyer, J. and Kohlmeyer, E. (1979). Marine Mycology-The Higher Fungi. (Academic Press, New York).

Krauss, G., Bärlocher, F., Schreck, P., Wennrich, R., Glässer, W. and Krauss, G.J. (2001). Aquatic hyphomycetes occur in hyperpolluted waters in Central Germany. Nova Hedwigia. 72: 419-428.

Luo, J., Yin, J.F., Cai, L., Zhang, K.Q. and Hyde, K.D. (2004). Freshwater fungi in Lake Dianchi, a heavily polluted lake in Yunnan, China. Fungal Divers. 16: 93-112.

Maltby, L. and Booth, R. (1991). The effect of coal-mine effluent on fungal assemblages and leaf breakdown. Water Res. 25 (3): 247-250.

McLaughlin, D.J., McLaughlin, E.G., Lemke, P.E. and eds. (2001). The Mycota VIIA and VIIB. Berlin: SpringerVerlag.

Mohamed, D.J. and Martiny, J.B. (2010). Patterns of fungal diversity and composition along a salinity gradient. ISME J 5 (3): 379-388.

Pascoal, C., Căssio, F. and Marvanova, L. (2005). Anthropogenic stress may affect aquatic hyphomycete diversity more than leaf decomposition in a low-order stream. Arch. Hydrobiol. 162: 481-496.

Pattee, E. and Chergui, H. (1995). The Application of Habitat Templets and Traits to Hyphomycete Fungi in a Mid-European River System. Freshwater Biol. 33 (3): 525-539.

Raja, H.A., Schmit, J.P. and Shearer, C.A. (2009). Latitudinal, habitat and substrate distribution patterns of freshwater ascomycetes in the Florida Peninsula. BiodiversConserv. 18 (2): 419-455.

Ranković, B. (2005). Five Serbian reservoirs contain different fungal propagules. Mycologia. 97 (1):50-56.

Raviraja, N.S., Sridhar, K.R. and Bärlocher, F. (1998). Fungal species richness in Western Ghat streams (southern India): is it related to $\mathrm{pH}$, temperature or altitude. Fungal Divers. 1: 179-191.

RNPD (1989). Water Quality Conditions in the River Nile System: A General Rev.

Roache, M.C., Bailey, P.C. and Boon, P.I. (2006). Effects of salinity on the decay of the freshwater macrophyte, Triglochinprocerum. Aquat. Bot. 84 (1): 45-52.

Shearer, C.A. (1972). Fungi of the Chesapeake Bay and its tributaries. III. The distribution of wood-inhabiting Ascomycetes and Fungi Imperfecti of the Patuxent River. Am. J. Bot.961969.

Sridhar, K.R. and Bärlocher, F. (1997). Water chemistry and sporulation by aquatic hyphomycetes. Mycol. Res. 101: 591596.

Suberkropp, K. (1984). Effect of temperature on seasonal occurrence of aquatic hyphomycetes. Trans. Brit. Mycol. Soci. 82: 53-62.

Suberkropp, K. and Chauvet, E. (1995). Regulation of Leaf Breakdown by Fungi in Streams-Influences of Water 
Chemistry. Ecology. 76 (5): 14331445.

Suberkropp, K., Michelis, A., Lorch, H.J. and Ottow, J.C. (1988). Effect of Sewage-

Treatment Plant Effluents on the Distribution of Aquatic Hyphomycetes in the River Erms, Schwabische Alb, F-R-G. Aquat. Bot. 32 (1-2): 141-153.

Tan, T. and Lim, G. (1984). A comparison of fungi from polluted water. Environmental Pollution Series A, Ecological and Biological. 35 (1): 5765.

Tsui, C.K., Hyde, K.D. and Hodgkiss, I.J. (2001). Longitudinal and temporal distribution of freshwater ascomycetes and dematiaceous hyphomycetes on submerged wood in the Lam Tsuen River, Hong Kong. J. N. Am. Benthol. Soc. 20 (4): 533-549.
Wainwright, M. (1988). Metabolic Diversity of Fungi in Relation to Growth and Mineral Cycling in Soil-a Review. Trans. Brit. Mycol Soc. 90:159-170.

Wong, K.M.K., Goh, T.K., Hodgkiss, I.J., Hyde, K.D. et al. (1998). Role of fungi in freshwater ecosystems. Biodiversity and Conservation 7, 1187-1206.

World Bank (2005). Country Environmental Analysis (1992-2002)" Arab Republic of Egypt, Water and Environment Department, the Middle East and North Africa Region. World Bank.

Wurzbacher, C.M., Bärlocher, F. and Grossart, H.P. (2010). Fungi in lake ecosystems. Aquat.Microb. Ecol. 59 (2): 125-149.

Yuen, T.K., Hyde, K.D., Hodgkiss, I.J. (1998). Physiological growth parameters and enzyme production in tropical freshwater fungi. Material und Organismen. 32, 2-6. 


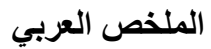

العلاقه بين الخواص الكيميائيه والفيزيائيه لتفرعات نهز النيل والتنوع الفطري في منطقه الالتا (مصر)

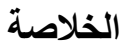
تلوث مياه نهر النيل في في مصر في ازدياد دائم مع استمر ار تدفق النفايات بشكل مستمر. عينات من المياه جمعت من ترع وتفر عات نهر النيل الاساسيه من ثمان محافظات في الدلتا علي مدار اربع مواسم مختلفه في الفتره من فبر اير 2010 الي ديسمبر 2011. العديد من العلاقات ما بين خو اص المياه الكيميائيه و الفيزيائيه و التتوع الفطري في منطقة الالتا تم دراستها.درجة حرارة الهواء و الماء كانت من اكثر العو امل الاساسيه الموثره علي توزيع الفطريات. تركيز الكالسيوم و المغنسيوم كان لها تاثير عكسي علي تواجد الفطريات بينما تواجد الكبريتات والنيتريت لم يكن لها تأثير واضح.الامونيا والفوسفات كان لهم تأثير محفز لتوزيع الفطريات في منطقة الدلتا. ملوحة المياه كان لها تأثير و اضح علي تحديد نوعية وتنوع الفطريات في منطقة 